\title{
SEPIVAC: a community-based study of stroke incidence in Umbria, Italy
}

\author{
Stefano Ricci, Maria Grazia Celani, Francesco La Rosa, Rino Vitali, Emilio Duca, \\ Remo Ferraguzzi, Marida Paolotti, Donatella Seppoloni, Nevia Caputo, Cesare Chiurulla, \\ Reana Scaroni, Enrico Signorini
}

\begin{abstract}
The SEPIVAC study is a communitybased epidemiological survey of incidence and outcome of acute cerebrovascular disease in the Sixth Local Health Unit, Umbria, Italy (population 49 218). The study was carried out from 1 September 1986 to 31 August 1989. There were 375 patients who were registered with a first ever stroke, with a crude rate of 2.54 (95\% confidence limits $2 \cdot 29-2 \cdot 81)$ per 1000 per year; the rate adjusted to the European population is 1.55 (CL 1.361.77). The age adjusted relative risk for males is 1.35 (CL $1 \cdot 10-1 \cdot 66$ ). Up to $15 \%$ of the patients were not admitted to hospital during the acute phase of their disease. At least $286(76.3 \%$, CL $72-80.6)$ of the cases were due to cerebral ischaemia; in 56 of these $(19.6 \%$, CL 15-24.2) a clinical diagnosis of lacunar ischaemia was made. The $\mathbf{3 0}$ day case fatality rate was $20 \cdot 3 \%$ (CL 16.2-24.3); between one and six months $7 \cdot 5 \%$ (CL 5-10.6) of patients died.
\end{abstract}

Perugia University Hospital, Perugia Clinica Neurologica S Ricci M Grazia Celani

Servizio di

Neuroradiologia

N Caputo

C Chiurulla

R Scaroni

E Signorini

Dipartimento di

Igiene, Perugia

University, Perugia

F La Rosa

$R$ Vitali

USL $n 6$ dell'Umbria, Panicale, Italy

E Duca

R Ferraguzzi

M Paolotti

D Seppoloni

Correspondence to: Dr Ricci, Clinica

Dr Ricci, Clinica

Pozzo, 06100 Perugia, Italy.

Received 13 August 1990

and in revised form

12 November 1990 .

Accepted 22 November 1990
Since stroke is by no means a single pathological entity, we must identify the outcome of pathologically homogeneous groups of patients, if we want to eventually find an effective treatment for this leading cause of death and disability. Community based studies, planned to measure the incidence and outcome of the different subgroups of stroke ${ }^{1}$ are thus required for this purpose. Since the results of these studies will be used to plan clinical trials which will probably be based in several centres and in different countries, it is important to obtain information from many areas of the world, and to look at differences and similarities which could be responsible for a particular impact of a given treatment.

The SEPIVAC study ${ }^{2}$ is the first community-based epidemiological survey of stroke incidence and outcome in Italy. It was planned taking into account the experience of the Oxfordshire Community Stroke Project, ${ }^{3}$ thus allowing close comparison of the results. The aim of the study was to determine the incidence of different types of first ever stroke in a representative area of Central Italy. We present here the final results on incidence, 30 day and six month case fatality rate of first ever stroke. A further paper will discuss the whole problem of outcome.

\section{Material and methods}

The SEPIVAC study (Studio Epidemiologico sulla Incidenza delle Vasculopatie Acute Cerebrali) was a community-based epidemiological survey of incidence and outcome of acute cerebrovascular disease in the Sixth Local Health Unit (USL 6, del Trasimeno), Umbria, Italy (fig 1).

The registration of patients began on $1 \mathrm{Sep}-$ tember 1986 and continued until 31 August 1989.

The study population comprised all residents in USL 6; we obtained data on residents on 31 December 1986-88 from the official authorities of the Umbria region (Centro Regionale Elaborazione Dati) and considered the sum of these figures as our population at risk; there were 49101,49220 and 49333 residents at each time, thus giving a mean of 49218 residents per year.

The methodology of our study has been described in detail elsewhere. ${ }^{2}$ In summary, cases were registered with the study in one of the following ways: (1) notification from the general practitioner; (2) on call medical service; (3) hospital admission registers of the three hospitals in the USL 6 territory and of the two hospitals in Perugia, the main town in Umbria, where a significant part of the Trasimeno population is usually admitted; (4) death certificates.

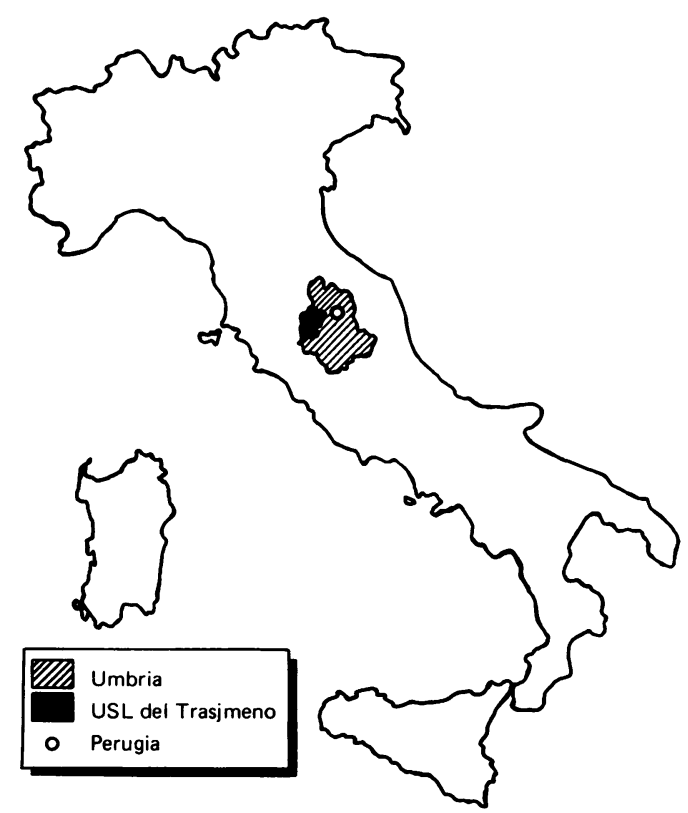

Figure 1 Geographical location of the study area. 
Table 1 Average age and sex specific incidence rates/1000 for first stroke

\begin{tabular}{|c|c|c|c|}
\hline Age & $\begin{array}{l}\text { No of cases/No at risk } \\
(\text { pop. } \times 3)\end{array}$ & Rate & $\begin{array}{l}95 \% \text { confidence } \\
\text { intervals }\end{array}$ \\
\hline $\begin{array}{c}\text { MALES: } \\
0-54 \\
55-64 \\
65-74 \\
75-84 \\
>85 \\
\text { Total }\end{array}$ & $\begin{array}{c}14 / 49 \cdot 463 \\
35 / 10 \cdot 359 \\
55 / 7 \cdot 264 \\
67 / 4 \cdot 101 \\
12 / 678 \\
183 / 71 \cdot 865\end{array}$ & $\begin{array}{r}0 \cdot 28 \\
3 \cdot 38 \\
7 \cdot 57 \\
16 \cdot 34 \\
17 \cdot 70 \\
2 \cdot 55\end{array}$ & $\begin{array}{r}0 \cdot 16-0.49 \\
2 \cdot 39-4.75 \\
5.76-9.93 \\
12 \cdot 76-20.88 \\
9.59-31.87 \\
2 \cdot 20-2.95\end{array}$ \\
\hline $\begin{array}{c}\text { FEMALI } \\
0-54 \\
55-64 \\
65-74 \\
75-84 \\
>85 \\
\text { Total }\end{array}$ & $\begin{array}{rr}12 / & 47.956 \\
25 / & 11.064 \\
32 / & 8 \cdot 831 \\
85 / & 6.322 \\
38 / & 1.616 \\
192 / & 75.789\end{array}$ & $\begin{array}{r}0 \cdot 25 \\
2 \cdot 26 \\
3.62 \\
13 \cdot 45 \\
23.51 \\
2 \cdot 53\end{array}$ & $\begin{array}{r}0.14-0.45 \\
1.49-3.39 \\
2.52-5 \cdot 18 \\
10 \cdot 80-16.71 \\
16 \cdot 87-32.63 \\
2 \cdot 19-2.92\end{array}$ \\
\hline
\end{tabular}

Any patient who might have had a cerebrovascular event was visited as soon as possible by one of us, either in hospital or at home. If a patient died very rapidly, or for any reason was not seen by the study group, we used all the available information from relatives, and from the GPs and hospital forms. We only registered with the study those patients whose clinical features fit the definition of transient ischaemic attack (TIA) or stroke that we had agreed to adopt. We met weekly to discuss each case before the definite registration of the patient. ${ }^{4}$ We attempted to obtain a CT scan within 30 days from the stroke in as many patients as possible. ECG, blood tests and Doppler examination of carotid arteries were also obtained whenever possible. All clinical and laboratory data were reported on a special form, including the Allen score for the clinical differentiation between ischaemia and haemorrhage ${ }^{5}$ and an estimate of pre-stroke handicap by means of a modified Rankin scale. ${ }^{6}$ Our cases were classified as follows: ${ }^{7}$ definite infarction (CT diagnosis); probable infarction (CT not carried out within 30 days, Allen score $<4)$; definite intracerebral haemorrhage (CT and/or LP diagnosis); probable intracerebral haemorrhage (CT not carried out within 30 days, Allen score $>24)$; definite subarachnoid haemorrhage (CT and/or LP diagnosis); probable subarachnoid haemorrhage (CT or LP not available, typical clinical picture); not known (CT not done within 30 days, Allen score not available or between four and 24). Probable and definite CI were further subdivided, according to a recently proposed clinical classification, ${ }^{89}$ into total anterior circulation syndromes (TACS), partial anterior circulation syndromes (PACS), lacunar syndromes (LACS), posterior circulation syndromes (POCS), and not known (NK).

Patients were followed prospectively: at one

Table 2 Average age specific incidence rates/1000 for first stroke

\begin{tabular}{lrrr}
\hline Age & $\begin{array}{l}\text { No of cases/No at risk } \\
\text { (pop. } \times 3)\end{array}$ & Rate & $\begin{array}{c}\text { 95\% confidence } \\
\text { intervals }\end{array}$ \\
\hline MALES + FEMALES: & & & \\
$0-54$ & $26 / 97 \cdot 419$ & $0 \cdot 27$ & $0 \cdot 18-0 \cdot 40$ \\
$55-64$ & $60 / 21.423$ & $2 \cdot 80$ & $2 \cdot 16-3 \cdot 63$ \\
$65-74$ & $87 / 16.095$ & $5 \cdot 41$ & $4 \cdot 36-6 \cdot 70$ \\
$75-84$ & $152 / 10 \cdot 423$ & $14 \cdot 58$ & $12 \cdot 40-17 \cdot 14$ \\
$>85$ & $50 / 2 \cdot 294$ & $21 \cdot 80$ & $16 \cdot 35-28 \cdot 98$ \\
Total & $375 / 147.654$ & $2 \cdot 54$ & $2 \cdot 29-2 \cdot 81$ \\
\hline
\end{tabular}

Table 3 Comparison of Allen score and CT results

\begin{tabular}{l}
\hline \\
\hline
\end{tabular}

month, six months and one year from the event, survivors were reassessed and information on disability and new events was recorded on a follow up form. If the patient died, all the available medical records were reviewed to establish the cause of death. Data were recorded on an ad hoc prepared computer program and analysed using standard statistical methods. Results are reported with 95\% confidence limits (CL).

\section{Results}

In this particular paper we present results on incidence, 30 days and six months case fatality rate of first ever stroke. After clinical assessment by a study physician, 379 patients were considered to have had a first ever stroke during the study period. Four cases, however, were later excluded: one patient had a subdural haematoma, one patient had cerebral metastases, and two patients had a primary cerebral tumour (glioma). We excluded all these cases, even though in two (a glioma and the case with metastases) the lesions were observed in cerebral areas apparently not related to clinical symptoms. Of the remaining 375, $334(89 \%$, CL 86-92) were seen by the study team, with a median delay of four days. Fifty eight patients $(15 \%$, CL 12-19) were treated at home by their GPs, and were not admitted to hospital during the acute phase of their disease. Age and sex specific incidence rates of first stroke are given in tables 1 and 2. The crude rate is 2.54 (CL 2.29$2 \cdot 81)$ per 1000 per year. The rates adjusted to the Italian and European populations are 1.81 (CL 1.6-2.04) and 1.55 (CL 1.36-1.77). The crude relative risk (RR) for males is 1.01 , whereas the Mantel-Haenszel weighted RR is 1.35 (CL 1.1-1.66).

A pathological diagnosis was possible in 261 patients $(70 \%, \mathrm{CL} 65-74)$ : in 259 a CT scan was obtained within 30 days from the stroke, and in the remaining two a diagnostic LP was available. No necropsy was carried out. The comparison of CT results with the Allen score (which was available in 233-261 cases) yielded a $97 \%$ accuracy (table 3 ). The distribution of pathological types is shown in table 4 . To summarise, cerebral infarction (CI) accounted

Table 4 First strokes: pathological classification

\begin{tabular}{lrr}
\hline Definite CI & 218 & $(58 \cdot 1 \%$, CI $53 \cdot 1-63 \cdot 1)$ \\
Probable CI & 68 & $(18 \cdot 1 \%$, CI $14 \cdot 2-22 \cdot 0)$ \\
Definite PICH & 33 & $(8 \cdot 8 \%$, CI $6 \cdot 1-12 \cdot 1)$ \\
Probable PICH & 4 & $(1 \cdot 1 \%$, CI $0 \cdot 3-2 \cdot 7)$ \\
Definite SAH & 10 & $(2 \cdot 7 \%$, CI $1 \cdot 3-4 \cdot 9)$ \\
Probable SAH & 5 & $(1 \cdot 3 \%$, CI $0 \cdot 4-3 \cdot 1)$ \\
Not known & 37 & $(9 \cdot 9 \%$, CI $7 \cdot 0-13 \cdot 3)$ \\
\hline
\end{tabular}

See text for abbreviations. 
Table 5 First strokes: 30 day case fatality rate

\begin{tabular}{llr}
\hline Total: & $76 / 375$ & $(20 \cdot 3 \%$ CL 16.2-24.3) \\
SAH: & $10 / 15$ & $(66 \cdot 7 \%$ CL 42.8-90.5) \\
PICH: & $14 / 37$ & $(37 \cdot 8 \%$ CL 22.5-55.2) \\
NK: & $24 / 37$ & $(64 \cdot 9 \%$ CL 49.5-80.2) \\
CI: & $28 / 286$ & $(9 \cdot 8 \%$ CL 6.6-13.8) \\
\hline
\end{tabular}

for more than $75 \%$ of first strokes, whereas $10 \%$ were primary intracerebral haemorrhages (PICH) and 4\% were subarachnoid haemorrhages (SAH); in 37 cases a pathological diagnosis was not possible (NK)

Out of 286 definite and probable CI, $14.7 \%$ (CL 10.6-18.8) were TACS, 55.9\% (CL 50.261.7) were PACS, $19.6 \%$ (CL 15-24.2) were LACS and $8.4 \%$ (CL 5.5-12.2) were POCS; we could not define $1.4 \%$ of cases (CL $0.4-3.5$ ) due to the lack of clinical details. These results are shown in fig 2. When comparing this classification with CT scan results, minimal variations happened (fig 2); however, taking into account the possibility that the scan did not show the actual lesion, but an asymptomatic one, we decided to adopt the first clinical classification.

At 30 day follow up, 76 patients had died $(20 \cdot 3 \%$, CL $16 \cdot 2-24 \cdot 3)$. The case fatality rate by pathological types is shown in table 5 . Out of 28 patients with CI who died, 20 had a TACS (case fatality rate $47 \cdot 6 \%$, CL 32-63.6), five had a PACS $(3 \cdot 1 \%$, CL $1-7 \cdot 2)$, two a POCS $(8.3 \%$, CL 1-27) and one was not classified $(25 \%, \mathrm{CL}$ $0 \cdot 6-80 \cdot 6)$; no patient with LACS died within 30 days.

Between one and six months 28 patients died (table 6); thus the case fatality rate at six month follow up was $27 \cdot 7 \%$ (CL 23.2-32.3). Among the 24 patients with ischaemic stroke who died between one and six months, seven had a TACS $(16.7 \%$, CL $7-31.4), 13$ had a PACS $(8 \cdot 1 \%$, CL $4 \cdot 4-13 \cdot 5)$, three had a LACS $(5 \cdot 4 \%$, CL $1 \cdot 1-14.9 \%)$ and one was not classified (25\%, CL 6.3-80.6); no patient with POCS died during this period.

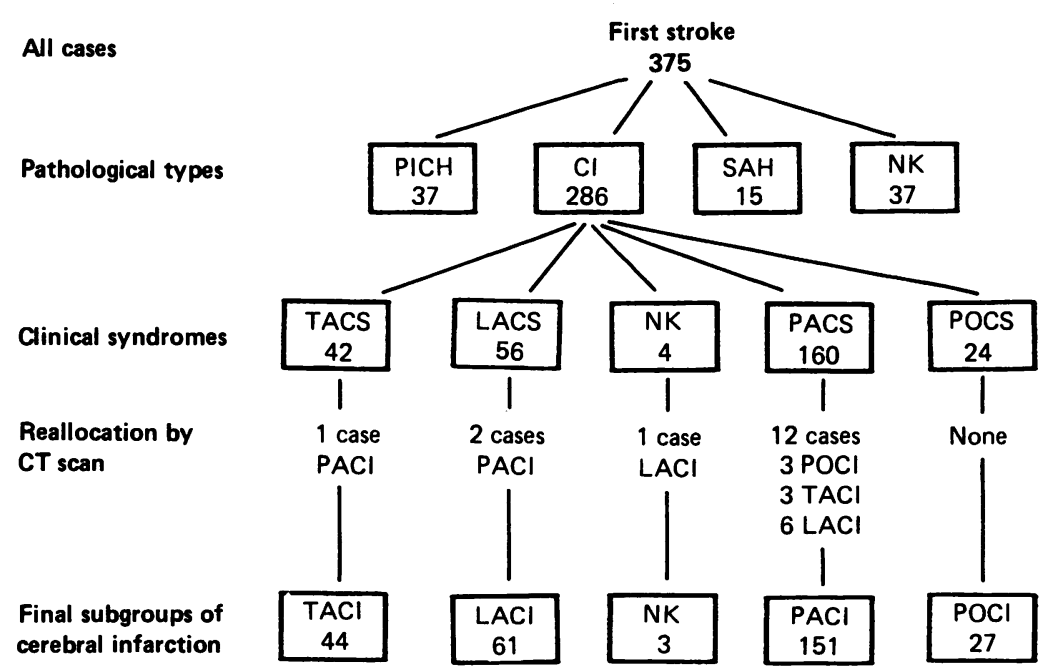

Figure 2 Clinical classification of cerebral infarction. PICH: primary intracerebral haemorrhage; CI: cerebral infarction; SAH: subarachnoid haemorrhage; NK: not known. TACS (I): Total anterior circulation syndrome (infarction). PACS (I):

Partial anterior circulation syndrome (infarction). LACS (I): Lacunar syndrome (infarction). POCS (I): Posterior circulation syndrome (infarction).
Table 6 First strokes: 6 month case fatality rate

\begin{tabular}{lrl}
\hline Total: & $104 / 375$ & $(27 \cdot 7 \%$ CL 23.2-32.3) \\
SAH: & $11 / 15$ & $(73 \cdot 3 \%$ CL 44.9-92.2) \\
PICH: & $15 / 37$ & $(40 \cdot 5 \%$ CL 24.7-57.9) \\
NK: & $26 / 37$ & $(70 \cdot 3 \%$ CL $53-84 \cdot 1)$ \\
CI: & $52 / 286$ & $(18 \cdot 2 \%$ CL $13 \cdot 7-22 \cdot 7)$ \\
\hline
\end{tabular}

\section{Discussion}

In this study only four out of 379 cases which had been diagnosed as "first ever in a lifetime stroke" on clinical grounds were found to have some other lesion on CT $(1.06 \%$, CL $0.03-$ $2 \cdot 1$ ); we can therefore confirm that the clinical diagnosis of stroke is highly reliable, ${ }^{10}$ especially taking into account the fact that in two of these cases it was very unlikely that the symptoms were due to the lesions shown by CT. Furthermore, this result makes us confident in excluding a non-vascular pathology in those patients who were assessed, but did not have a CT scan.

Although our crude incidence rate is quite high, our results do not differ from those of other studies, when adjusting for the age distribution of our population: in fact, rates adjusted to the European population are 1.55 per 1000 per year for SEPIVAC and 1.56 per 1000 per year for OCSP. ${ }^{3}$ The rate of stroke incidence increases with increasing age, as shown in all the "ideal" studies of stroke epidemiology. ${ }^{1}$

After adjusting for age, we found a $35 \%$ excess of risk of stroke for males, which is statistically significant; interestingly, this risk does not appear in the crude comparison, due to the older age of our female population.

We found that $15 \%$ of our patients were never admitted to hospital during the acute phase of their disease. The proportion of stroke patients admitted to hospital varies widely in different countries, as well as in different regions of the same country, depending on hospital facilities, tradition, community services, and willingness of GPs to treat these patients at home. The proportion of "home" patients rose during the study period, probably because GPs were aware of the fact that we offered CT scan and Doppler facilities-including ambulance transport-as a part of the project; nevertheless, this figure appears unusual for Italy, where, as in Canada, ${ }^{11}$ virtually all patients with stroke are said to be admitted to hospital within a few hours. This fact must be borne in mind when planning clinical trials of stroke treatment, as well as secondary prevention studies. It is our current view that there is no need to encourage hospital admission, since we cannot offer an effective treatment for stroke; instead, a community-based outpatient clinic with special expertise in cerebrovascular disease might improve both the rate of detection and the quality of treatment of these patients. In the meantime, a hospital-based stroke unit can be set up, to run trials of acute treatment.

It is well known that it is not possible to reliably differentiate $\mathrm{CI}$ and PICH on clinical grounds only. ${ }^{12}$ For this reason, we tried to obtain a CT scan whenever possible, and consider a $\mathbf{7 0 \%}$ rate a very good result; no 
necropsy was performed, due to local tradition. When our study was planned, in order to make a differential diagnosis between infarction and haemorrhage, it was considered safe to obtain a CT within 30 days from the stroke. This statement was recently challenged, ${ }^{13}$ and a period of 15 days considered safer. However, in community-based studies of stroke incidence, the 30 day limit can be considered a reasonable goal. ${ }^{14}$ The classification of pathological types of strokes was achieved by means of the Allen score in $19 \%$ of our cases; taking into account the high accuracy of this score, and the epidemiological purpose of the study, we consider that we have introduced a very small bias, if any, with its use. Ten per cent of strokes were not defined from a pathological point of view, yet we think that this is a reasonable price to pay for the unvoidable organisation difficulties of studies like ours.

Up to $76 \%$ of our cases were due to cerebral ischaemia; according to the clinical classification we adopted, ${ }^{89} 56$ out of $286(19.6 \%$, CL 15-24.2) ischaemic strokes were due to lacunar infarctions. This figure is similar to that in other studies. ${ }^{15}$ We did not find any LACS in haemorrhagic strokes, whereas we found 18 TACS and 17 PACS in patients with PICH; from these results we conclude that the clinical diagnosis of lacunar ischaemia can be made with confidence.

The 30 day case fatality rate was lower than those reported in hospital series, and this is almost certainly due to the fact that we have also collected very mild cases, treated both at home or in hospital, just for a few days. These figures appear very similar to those observed in the OCSP ${ }^{7}$ a higher case fatality rate has been described in Tilburg, ${ }^{16}$ but in that study all strokes, and not only first ever in a lifetime strokes, were included. It is interesting to note that TACS due to CI have a higher case fatality rate than PICH, and this is probably due to the fact that we have been able to register even the small haemorrhages, which do not cause death by herniation during the first week after the stroke. Finally, no patient with LACS died within the first month, and only three out of 56 within six months. This fact clearly stresses the importance of separating this form of cerebral ischaemia in trials of both secondary prevention and acute treatment of ischaemic stroke.

We thank Professor Charles Warlow, Edinburgh, Dr Peter Sandercock, Edinburgh, and Dr John Bamford, Leeds, for their Sandercock, Edinburgh, and Dr John Bamford, Leeds, for their help in our study. We also thank Mr Italo March
very useful work in the computer data storing.

The study was partly supported by Regione dell' Umbria Assessorato Sanita, and USL n.6 of Umbria.

1 Malgren R, Warlow C, Bamford J, Sandercock P. Geographical and secular trends in stroke incidence. Lance 1987;ii:1196-200.

2 Ricci S, Celani MG, Guercini G, et al. First-year results of a Community-based study of stroke incidence in Umbria, Italy. Stroke 1989;20:853-7.

3 Bamford J, Sandercock P, Dennis M, et al. A prospective study of acute cerebrovascular disease in the community: the Oxfordshire Community Stroke Project 1981-86. 1 . Methodology, demography and incident cases of first-ever Methodology, demography and incident cases of first-ever
stroke. J Neurol Neurosurg Psychiatry 1988;51:1373-80.

stroke. J Neurol Neurosurg Psychiatry 1988;51:1373-80.
4 Oxfordshire Community Stroke Project. Incidence of stroke in Oxfordshire: first year's experience of a community stroke register. $B M J$ 1983;287:713-7.

5 Allen C. Clinical diagnosis of the acute stroke syndrome. $Q J$ Med 1983;8:515-23.

6 Rankin J. Cerebrovascular accidents in patients over 65 years of age: prognosis. Scott Med J 1957;2:200-15

7 Bamford J, Sandercock P, Dennis M, Burn J, Warlow C. A prospective study of acute cerebrovascular disease in the community: the Oxfordshire Community Stroke Project 1981-86. 2. Incidence, case fatality rates and overall outcome at one year of cerebral infarction, primary outcome at one year of cerebral infarction, primary
intracerebral and subarachnoid haemorrhage. $J$ Neurol intracerebral and subarachnoid haem

8 Bamford J. The classification and natural history of acute cerebrovascular disease (thesis). University of Manchester, 1986.

9 Bamford J (for OCSP) and Ricci S (for SEPIVAC): Subgroups of cerebral infarctions in two community studies. J Neurol 1990;237:136 (abst.).

10 Sandercock P, Molineaux A, Warlow C. Value of computed tomography in patients with stroke: The Oxfordshire Community Stroke Project. BMJ 1985;290:193-7.

11 Abu-Zheid H, Choi N, Nelson N. Epidemiologic features of cerebrovascular disease in Manitoba: incidence by age, sex, and residence with etiologic implications. Can Med Ass J 1975;113:379-84.

12 Harrison MJG. Clinical distinction of cerebral haemorrhage and cerebral infarction. Postgrad Med J 1980;56:629-32.

13 Dennis M, Bamford J, Molineaux A, Warlow C. Rapid resolution of signs of primary intracerebral haemorrhage in computed tomograms of the brain. $B M J 1987 ; 95$ : 379-81.

14 Savoiardo $M$, Bracchi $M$. Neuroradiology of stroke. In: Callaghan N, Galvin R, eds. Recent research in neurology. London: Pitman, 1984.

15 Bamford J, Sandercock P, Jones L, Warlow C. The natura history of lacunar infarction: the Oxfordshire Community Stroke Project. Stroke 1987;18:545-51.

16 Herman B, Leyten ACM, Van Luijk JH, Frenken CWGM, De Coul O, Schulte BPM. Epidemiology of stroke in Tilburg, The Netherlands. The population-based stroke incidence register: 2 . Incidence, initial clinical picture and medical care, and three week case fatality. Stroke 1982;13: 334-9. 